\title{
Two-Particle Resonant States in a Many-Body Mean Field
}

\author{
R. Id Betan, ${ }^{1,2}$ R. J. Liotta, ${ }^{1}$ N. Sandulescu, ${ }^{1,3,4}$ and T. Vertse ${ }^{1,5}$ \\ ${ }^{1}$ Royal Institute of Technology, SCFAB, SE-10691, Stockholm, Sweden \\ ${ }^{2}$ Departamento de Fisica, FCEIA, UNR, Avenida Pellegrini 250, 2000 Rosario, Argentina \\ ${ }^{3}$ Institute of Physics and Nuclear Engineering, P.O. Box MG-6, Bucharest-Magurele, Romania \\ ${ }^{4}$ Research Center for Nuclear Physics, Osaka University, Osaka, Japan \\ ${ }^{5}$ Institute of Nuclear Research of the Hungarian Academy of Sciences, H-4001 Debrecen, P.O. Box 51, Hungary \\ (Received 24 January 2002; published 3 July 2002)
}

\begin{abstract}
A formalism to evaluate the resonant states produced by two particles moving outside a closed shell core is presented. It is found that long lived two-body states (including bound states) are mostly determined by either bound single-particle states or by narrow single-particle resonances. However, they can be significantly affected by the continuum part of the spectrum.
\end{abstract}

DOI: 10.1103/PhysRevLett.89.042501

The prospect of reaching and measuring very unstable nuclei, as is materializing now, opens the possibility of studying spectroscopic processes occurring in the continuum part of nuclear spectra. Much work has already been done in this subject, particularly regarding halo nuclei [1]. Still, the role played by single-particle resonances and of the continuum itself upon particles moving in the continuum of a heavy nucleus is not fully understood.

In this Letter we approach this problem by using the single-particle representation developed in Ref. [2] (Berggren representation). One chooses the proper continuum as a given contour in the complex energy plane and forms the basis set of states as the bound states plus the Gamow resonances included in that contour plus the scattering states on the contour [2].

Using the Berggren representation one can evaluate any one-particle quantity in the complex energy plane, e.g., the eigenstates of a deformed potential in terms of the Berggren states provided by a spherical basis. One may thus think that the Berggren representation can also be used straightaway to evaluate many-particle quantities, as one does with the shell model using bound representations. Unfortunately this is not the case. The root of the problem is that the set of energies of the two-particle basis states may cover the whole complex energy plane of interest. To show this we analyze the relatively simple case of two particles outside a core in terms of the Green function.

The two-particle Green function is $G_{2}(E)=[1+$ $\left.G_{2}^{(0)}(E) v_{\text {eff }}\right]^{-1} G_{2}^{(0)}(E)$, where $G_{2}^{(0)}$ is the bare (zerothorder) two-particle Green function given by [3]

$$
G_{2}^{(0)}\left(r, r^{\prime} ; E\right)=\sum_{i \leq j=1}^{N} \frac{\varphi_{i}(r) \varphi_{j}(r) \varphi_{i}\left(r^{\prime}\right) \varphi_{j}\left(r^{\prime}\right)}{E-\left(\epsilon_{i}+\epsilon_{j}\right)} .
$$

In our case the functions $\varphi_{j}(r)$ are members of the Berggren representation. By choosing an arbitrary path for the scattering states belonging to this representation one may obtain a continuum plane of zeroth-order poles corresponding to the sum $\epsilon_{i}+\epsilon_{j}$. Therefore, in this plane one will not be able to evaluate the infinite number
PACS numbers: 21.60.Cs, 23.50. $+\mathrm{z}, 24.30 . \mathrm{Gd}, 25.60 .-\mathrm{t}$

of two-particle poles which would be immersed in the continuum set of zeroth-order poles.

As an illuminating example we show in Fig. 1(a) a rectangular one-particle contour without any discrete state. From Eq. (1) one sees that in the complex two-particle energy plane the zeroth-order poles are given by the geometrical sum of a point $i$ on the one-particle contour (corresponding to the complex energy $\epsilon_{i}$ ) plus another point $j$ on that contour, such that $i \leq j$. These energies are located in the two-particle complex energy plane as shown in Fig. 1(b).

We thus see that if $2 a<b$ then there is a region in the two-particle complex energy plane which is free from any uncorrelated solution. Therefore, in this region one can search for resonant states of the interacting twoparticle system. By choosing the real energies $a$ and $b$ conveniently, i.e., such that $2 a<b$ as in Fig. 1(b), one

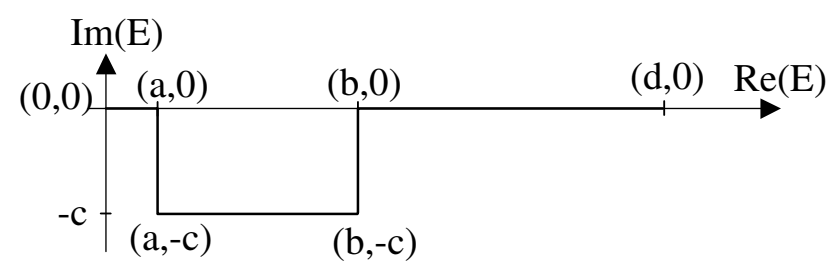

(a)

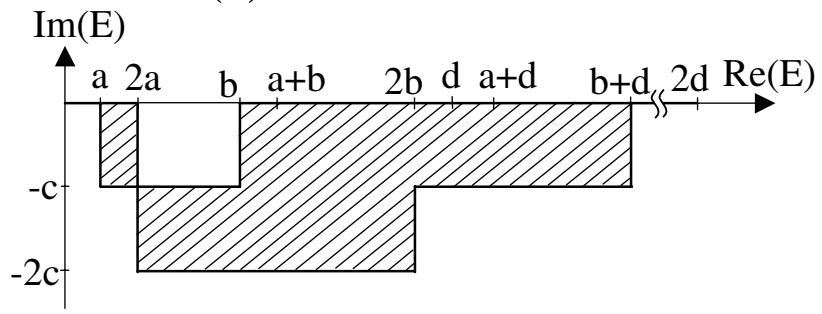

(b)

FIG. 1. (a) The points in this contour define the scattering functions that form the representation to be used in the twoparticle basis. (b) Two-particle energy plane where all points are zeroth-order energy solutions (dashed region). The white area corresponds to the allowed region. 
can study two-particle resonances lying in any reasonable energy region. We call this the "allowed" energy region.

Using the Berggen representation one can obtain the two-particle Tamm-Dancoff approximation equations in a standard fashion. We use for $v_{\text {eff }}$ a separable force so that those equations convert into the usual dispersion relation [4].

To generate the single-particle states we use a WoodsSaxon (WS) potential. The field $f_{\lambda}(r)$ in the separable interaction is the derivative of the WS.

We apply the formalism presented here to analyze neutron excitations in a nucleus that would lie on or even beyond the drip line. That is, the Fermi level may be close to or even immersed in the continuum. We analyze these two possibilities separately.

The WS to be used corresponds to the double closed shell nucleus ${ }^{78} \mathrm{Ni}$. The parameters for the volume part of the interaction are $V_{0}=40 \mathrm{MeV}, r_{0}=1.27 \mathrm{fm}, a=$ $0.67 \mathrm{fm}$. The spin-orbit interaction has the same values of $r_{0}$ and $a$, but the depth of the potential is $V_{\text {so }}=$ $21.43 \mathrm{MeV}$. With these parameters one obtains two bound states and four low-lying relatively narrow Gamow resonances, namely (energies in $\mathrm{MeV}), \epsilon_{1 d_{5 / 2}}=-0.800$, $\epsilon_{2 s_{1 / 2}}=-0.284, \quad \epsilon_{1 d_{3 / 2}}=(1.325,-0.479), \quad \epsilon_{0 h_{11 / 2}}=$ $(3.296,-0.013), \quad \epsilon_{1 f_{7 / 2}}=(3.937,-1.796)$, and $\epsilon_{0 g_{7 / 2}}=$ $(4.200,-0.167)$. These single-particle states are quite similar to the ones given by Skyrme-Hartree-Fock calculations [5]. We have checked that the shell $N=50$ is well defined, since there is a gap of about $3.6 \mathrm{MeV}$ between the lowest particle state, which here is $1 d_{5 / 2}$, and the highest hole state, i.e., $0 g_{9 / 2}$.

We also evaluate a case where no bound single-particle states are present. For this, we reduced the value of the depth of the WS to $V_{0}=37 \mathrm{MeV}$. With this potential the single-particle energies become $\epsilon_{1 d_{5 / 2}}=(0.294,-0.018)$, $\epsilon_{1 d_{3 / 2}}=(1.905,-1.241), \epsilon_{0 h_{11 / 2}}=(4.681,-0.069), \epsilon_{1 f_{7 / 2}}=$ $(4.455,-2.851)$, and $\epsilon_{0 g_{7 / 2}}=(5.799,-0.506)$. There is not any bound state and the Gamow state $s_{1 / 2}$ has disappeared, as expected for neutron excitations. The effect of this state, if any, would be taken care of by the corresponding scattering functions.

We here present two-particle states with angular momentum $\lambda=0$, for which the separable force is known to reproduce well experimental data when available. We first analyze the case where there are bound singleparticle states. To determine the strength $G_{\lambda}$ of the separable force we follow the standard procedure of adjusting it by fitting the energy of a two-particle state, which usually is experimentally known. In our case we assume that such a state, which would be the ground state of ${ }^{80} \mathrm{Ni}$, exists below twice the energy of the lowest single particle state with an energy gap $\Delta=1.4 \mathrm{MeV}$, i.e., the ground state energy is $-3 \mathrm{MeV}$. For the scattering states we used a rectangular contour with the vertices as in Fig. 1(a) with $a=0.5 \mathrm{MeV}, b=9 \mathrm{MeV}, c=-4 \mathrm{MeV}$, and $d=20 \mathrm{MeV}$. We thus include in the Berggren basis all the bound and Gamow states given above for this case. The allowed region, therefore, comprises the two-particle energy plane with complex energies $\left(E_{r}, E_{i}\right)$ such that $1 \mathrm{MeV}<E_{r}<9 \mathrm{MeV}$ and $-4 \mathrm{MeV}<E_{i}<0 \mathrm{MeV}$.

As in Ref. [2] we use a Gaussian method of integration over the contour. The corresponding Gaussian points provide the scattering waves constituting the basis elements on the continuum. We have found that in order to obtain convergence within six digits in the evaluated quantities, one has to include ten Gaussian points for each $\mathrm{MeV}$ on the lines of the contour, except for the last segment [the one going from $(b, 0)$ to $(d, 0)]$ where five points for each $\mathrm{MeV}$ is enough. We arrive at this conclusion by always choosing the contour such that the resonances lie at least $300 \mathrm{keV}$ from the borders of the contour. The number of scattering states thus included in the basis is $N_{g}=225$. In Table I we show the convergence of the results as a function of $N_{g}$ as well as the influence of the continuum upon the calculated states.

One can check the reliability of the results by performing a calculation over the real energy axis only [3]. The real (bound) energies thus obtained, which we call "exact," should coincide with those evaluated by using any contour. Moreover, the value of $G_{\lambda}$ should, in all cases, be a real quantity. All these requirements are indeed fulfilled in our calculations.

In Fig. 2 we present all the calculated energies which we found inside the allowed region of the complex twoparticle plane. The first feature that strikes the eye in this figure is the straight line pattern that follow most of the

TABLE I. Energies (in MeV) of the $\lambda=0$ first excited bound state and of the lowest two-particle resonances in ${ }^{80} \mathrm{Ni}$. The energies are given as a function of the number of scattering states included in the single-particle representation, i.e., the number $N_{g}$ of Gaussian points. For $N_{g}=0$ the representation consists of bound states and Gamow resonances only. The calculation of these states was performed by using the high precision piecewise perturbation method [6].

\begin{tabular}{rcccc}
\hline \hline$N_{g}$ & $E_{1}$ & $E_{2}$ & $E_{3}$ & $E_{4}$ \\
\hline 0 & $(-0.642,0.012)$ & $(2.158,0.719)$ & $(3.268,-0.883)$ & $(7.931,-0.198)$ \\
35 & $(-0.65417,0)$ & $(1.96874,-0.39235)$ & $(3.92420,-1.05208)$ & $(7.95693,-0.25236)$ \\
70 & $(-0.65274,0)$ & $(1.96988,-0.39321)$ & $(3.92429,-1.05159)$ & $(7.95691,-0.25251)$ \\
110 & $(-0.65274,0)$ & $(1.97261,-0.39838)$ & $(3.92416,-1.05168)$ & $(7.95687,-0.25250)$ \\
225 & $(-0.65308,0)$ & $(1.97241,-0.39935)$ & $(3.92390,-1.05189)$ & $(7.95685,-0.25249)$ \\
550 & $(-0.65308,0)$ & $(1.97241,-0.39935)$ & $(3.92390,-1.05189)$ & $(7.95685,-0.25249)$ \\
\hline \hline
\end{tabular}




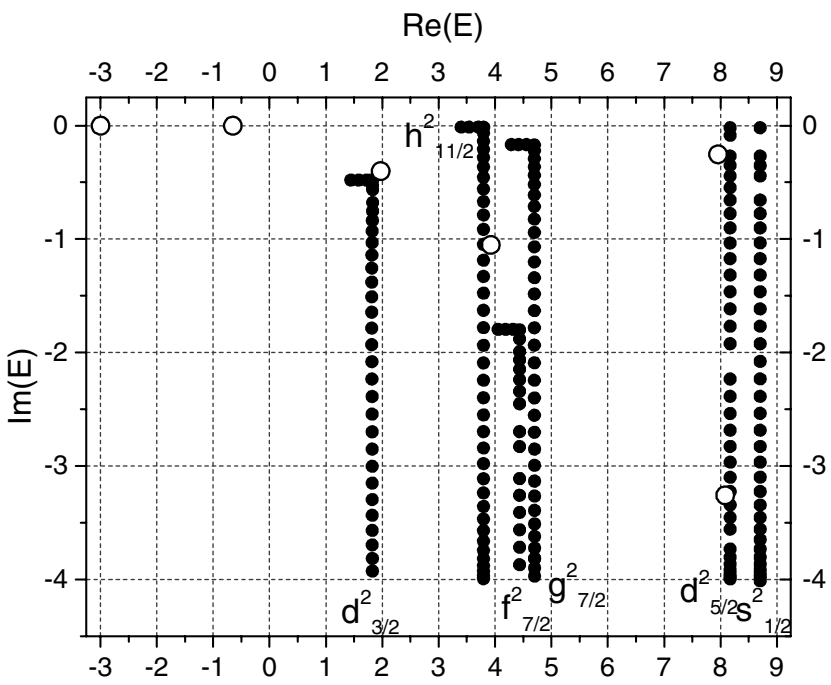

FIG. 2. Poles in the two-particle energy plane corresponding to states $\lambda=0$ in ${ }^{80} \mathrm{Ni}$. The labels of the straight lines correspond to configurations in which one of the two particles is in a bound state or a Gamow resonance and the other is in a scattering state. Energies are in $\mathrm{MeV}$.

energy points. These lines correspond to the basis states where one of the particles moves in a bound or Gamow state and the other in a fragment of the one-particle contour. For instance, the straight line at a real energy of $3.796 \mathrm{MeV}$ corresponds to a particle in the Gamow state $0 h_{11 / 2}$, with an energy $(3.296,-0.013) \mathrm{MeV}$, while the other is in the $h_{11 / 2}$ scattering states lying on the border at $a=0.5 \mathrm{MeV}$ in the contour of Fig. 1(a). The sum of both single-particle energies yields a real part of $3.796 \mathrm{MeV}$, which shows that these states are in fact poles of the zeroth-order Green function. Similar structures are found for all the straight lines in this figure, with the single-particle quantum numbers as indicated in the end of the lines. Thus, the horizontal segment at $-0.479 \mathrm{MeV}$ corresponding to the configuration $d_{3 / 2}^{2}$ is produced by a particle in the Gamow state $1 d_{3 / 2}$ while the other is on the scattering states belonging to the segment of the contour on the real energy axis between 0 and $a=0.5 \mathrm{MeV}$. This horizontal segment does not appear in the lines labeled $d_{5 / 2}^{2}$ and $s_{s / 2}^{2}$ in Fig. 2 because these lines are generated by the bound singleparticle states coupled to the scattering states on the border of the contour lying between $(b, 0)$ and $(b,-c)$ in Fig. 1(a).

We found that all the lines in Fig. 2 correspond to zeroth-order poles. Therefore the states on the lines are solutions of both the correlated and the uncorrelated twoparticle Hamiltonian. They do not describe the physical resonances that we are searching and can be considered spurious states. This peculiar feature of the continuum is also found in the one-particle case, where the states lying on the contour are solutions of both the correlated and the uncorrelated one-particle Hamiltonian, as was shown in Ref. [2].
Besides these peculiar lines the only two-particle states inside the allowed region are those indicated by open circles. These states, and the two bound ones at -3 and $-0.653 \mathrm{MeV}$, are mainly generated by configurations where both particles occupy bound states and/or Gamow resonances. Yet, the continuum part of the spectrum plays also an important role. In particular, one can see in Table I that the energies evaluated by excluding the scattering states do not fit well the correct results. It is also important to mention that in our calculations we require the Hamiltonian to be Hermitian, which implies that the strength $G_{\lambda}$ has to be a real quantity. But by fitting the energy of ${ }^{80} \mathrm{Ni}$ (gs) to the value $-3 \mathrm{MeV}$ and excluding the continuum, $G_{\lambda}$ becomes complex. By taking the corresponding real part only, as was done in Table I, that ground state energy acquires the unphysical value $(-2.856,0.359) \mathrm{MeV}$. Even the energy of the (bound) first excited state $E_{1}$ is unphysical since its energy is not real and the first resonance, i.e., $E_{2}$, is unphysical because the imaginary part of the energy is positive. But, as seen in Table I, a rather small number of scattering states is enough to obtain reasonable values for the energies. Thus, at $N_{g}=35$ one already reaches a precision of the order of a few keV.

The influence of $v_{\text {eff }}$ upon the two-particle resonances will be analyzed for the case where all single-particle states (and therefore the Fermi level) lie in the continuum. The single-particle resonances are wider than before, and therefore we used here a different one-particle contour, namely, $a=0.1 \mathrm{MeV}, b=13 \mathrm{MeV}, c=-6 \mathrm{MeV}$, and $d=26 \mathrm{MeV}$.

The straight lines discussed above appear also in this case with the same characteristics as before. To show the effect of the interaction upon the remaining states, which are the physical ones, we present in Fig. 3 the corresponding energies as a function of $G_{\lambda}$.

There is in this case only one two-particle bound state which materializes only when the interaction is large enough. This occurs in Fig. 3 at $G=0.023 \mathrm{MeV}$, where that bound state appears at an energy of $-0.104 \mathrm{MeV}$. The main components of the corresponding wave function are $(-0.95,0.03)\left(1 d_{5 / 2}\right)^{2}+(0.21,0.00)\left(0 h_{11 / 2}\right)^{2}+$ $(-0.17,0.07)\left(1 d_{3 / 2}\right)^{2}+(-0.13,0.01)\left(0 g_{7 / 2}\right)^{2}+(0.11$, $-0.05)\left(1 f_{7 / 2}\right)^{2}$. The interesting point is that this wave function does not change much as the interaction is increased, which shows the role played by the Gamow states in building up the bound states. The importance of these resonances is related to their widths. The wider the Gamow resonance the smaller is their influence. However, one cannot conclude from this that only Gamow resonances would be enough to describe the two-particle states of interest, since the inclusion of the complex contour is important to obtain even the narrow resonances and the bound states. In particular, without the contour the imaginary part of the energy corresponding to bound states becomes large, as happened in the previous example. 


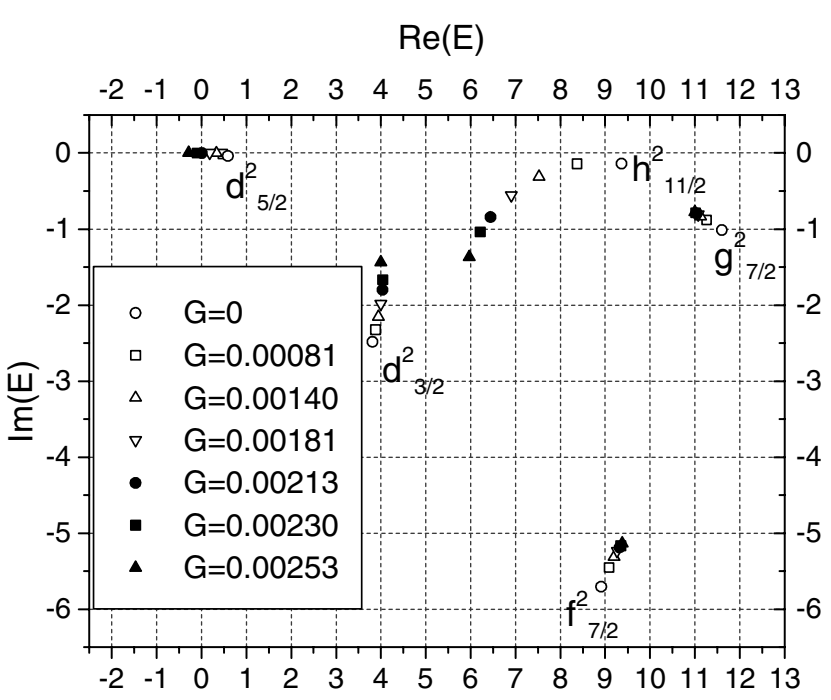

FIG. 3. Poles in the two-particle energy plane corresponding to the case where the Fermi level lies in the continuum. The value of the strength $G_{\lambda}$ (in $\mathrm{MeV}$ ) was chosen as explained in the text. The energies $\left(E_{r}, E_{i}\right)$ defining the allowed region are constrained to the values $0.2 \mathrm{MeV}<E_{r}<13 \mathrm{MeV}$ and $-6 \mathrm{MeV}<E_{i}<$ $0 \mathrm{MeV}$. The labels in each group of levels indicate the zerothorder configuration (i.e., at $G=0$ ) corresponding to the group. Energies are in $\mathrm{MeV}$.

An important conclusion that can be drawn from Fig. 3 is that due to the two-particle interaction wide resonances can give rise to narrow ones. This is shown by the states $g_{7 / 2}^{2}$ and $d_{3 / 2}^{2}$. But the surprising feature in Fig. 3 is that all the states become narrower as the interaction increases, except the state that in zeroth order is the narrowest one, i.e., $h_{11 / 2}^{2}$. This shows that, against expectations, the inclusion of only narrow single-particle states in the basis may not be a good starting point for the calculations, specially if the scattering functions corresponding to wide resonances are also excluded. This rather bizarre feature is a consequence of the Berggren metric, which makes that the matrix representation of the Hamiltonian is not Hermitian in the complex energy sector. Instead the bound state behaves in a standard shell model fashion, as expected for bound states.

In conclusion we have presented in this Letter a method to study two-particle resonances. This method is an extension of the shell model to the complex energy plane. We have shown that wide resonances and even the continuous background can be important to describe narrow two-particle resonances. These features are provided by the formalism in a straightforward fashion, i.e., by just analyzing the calculated two-particle energies and corresponding wave functions. This is an important point which is a characteristic of the shell model and which cannot be found in other methods used at present to study the continuum. Thus, formalisms which use real representations do not have proper (i.e., outgoing) boundary conditions, and therefore it is difficult to deal with resonances that do not correspond to quasibound states. In particular, one cannot analyze the evolution of wide resonances, as we have done above, because they show a very weak trace (or no trace at all) on the real energy axis [5]. The complex scaling method (CSM) [7,8] can be applied only when using dilation analytic central potentials for which the $\mathrm{ABC}$ theorem is valid [9]. In a Woods-Saxon potential the ABC theorem implies that one is not allowed to perform rotations beyond a certain critical angle; i.e., the CSM cannot treat resonances wide enough. Even the inclusion of narrow resonances can be a difficult undertaking within the CSM [8]. Moreover, in this method one rotates just the real energy axis, which thereby becomes a straight line in the complex energy plane. This implies that one has no control over the resonances that one considers in the calculation, since the resonances that can be included are those and only those that lie within the circular segment span by the rotation.

This work has been supported by FOMEC (Argentina), by the Hungarian OTKA fund No. T26244, No. T37991, and No. T29003, by the Swedish Foundation for International Cooperation in Research and Higher Education (STINT), and by the Swedish Institute.

Note added.- After submitting our Letter, the Letter by Michel et al. [10] was brought to our attention.

[1] F. M. Marqués et al., Phys. Rev. C 64, 061301(R) (2001), and references therein.

[2] R. J. Liotta, E. Maglione, N. Sandulescu, and T. Vertse, Phys. Lett. B 367, 1 (1996).

[3] G. F. Bertsch and H. Esbensen, Ann. Phys. (N.Y.) 209, 327 (1991).

[4] A. Evans et al., Nucl. Phys. A93, 261 (1967).

[5] N. Sandulescu, N. Van Giai, and R. J. Liotta, Phys. Rev. C 61, 061301(R) (2000).

[6] L. Gr. Ixaru, Numerical Methods for Differential Equations (Reidel, Dordrecht, 1984); L. Gr. Ixaru, M. Rizea, and T. Vertse, Comput. Phys. Commun. 85, 217 (1995).

[7] T. Myo, K. Kato, S. Aoyama, and K. Ikeda, Phys. Rev. C 63, 054313 (2001).

[8] R. Richler et al., Nucl. Phys. A618, 55 (1997).

[9] J. Aguilar and J. M. Combes, Commun. Math. Phys. 22, 269 (1971); E. Balslev and J. M. Combes, ibid. 22, 280 (1971).

[10] N. Michel, W. Nazarewicz, M. Płoszajczak, and K. Bennaceur, following Letter, Phys. Rev. Lett. 89, 042502 (2002). 